\title{
Analysis of the Binge Eating in China
}

\author{
Hui Feng ${ }^{1, a}$ \\ ${ }^{1}$ The Harley School Rochester, NY 14450
}

\begin{abstract}
Binge Eating Disorder(BED) is a common disorder of eating behavior. It is estimated to affect 1$3 \%$ of the total population. The research on binge eating is still in its early stages. The ubiquity of it made it important to explore the specific link between binge eating and negative emotions, especially among Chinese adolescents. BED is also common in China. There are different results of studies in other countries due to cultural, dietary, and psychological factors. This article explores the relationship between psychological factors (e.g., depression, anxiety, stress) and binge eating behavior among Chinese adolescents and how to deal with them.
\end{abstract}

\section{Introduction}

According to DSM-IV and DSM-5, there are five diagnostic criteria for binge-eating disorder. Individuals must report recurrent episodes of binge eating, which is characterized by eating an unusual amount of food. Most people eat at similar times and in similar circumstances for lack of control over eating during binge-eating time. Moreover, individuals have to experience at least three situations of the following: eating rapidly, eating until feeling uncomfortably full, eating large amounts of food when not feeling hungry, and eating alone because of being embarrassed by how much one is eating, or feeling depressed after overeating. The criteria also require evaluating patients' distress regarding binge eating. It has to occur on average at least two days a week for six months. Finally, BED cannot be associated with the frequent use of inappropriate compensatory behaviors (such as fasting or excessive exercise), nor can it occur during the onset of bulimia nervosa or anorexia nervosa.

According to the frequency of BED and improper compensation, BED was further classified into four grades: mild, moderate, severe, and extreme. During one week, the average frequency of binge eating and improper compensation was 1 to 3 times per week as mild, 4 to 7 times as moderate, 8 to 13 times as severe, and more than 14 times as extreme. The average frequency of binge eating and improper compensation was more than 2 times per day. [1] BED can affect anyone, regardless of age, race, gender, or any other demographic category. As overeating increases, individuals may begin to experience physical symptoms that negatively affect their lives such as skin problems, diarrhea, high blood pressure, and etc. Adolescents are the main group of people with eating disorders and are associated with physical, psychological, and social maturity in adolescents. In a study of US adolescents, the incidence rate for binge eating was 10.1 per 1000 person-years among females and 6.6 per 1000 person-years among males (both sexes $\geq 14$ years). [2] A study in South Korea shows that 28 percent of 327 female high school students showed a tendency to overeat.[3]

\section{Previous study}

While there are not many large prospective studies on $\mathrm{BED}$, a variety of factors for binge eating have been proposed. Binge eating is actually related to dieting. Dieters gained more weight than non-dieters, especially among girls. Because of over-concerned about body weight, more girls tend to binge eating than boys.[8] Similarly, adolescent boys and girls have different body change strategies, with girls more likely using strategies to lose weight and boys more likely using strategies to increase muscle tone (but not gain weight). [10] Although medically directed weight control may be beneficial for overweight teens, the data show that for many teens, dieting does not control weight and promotes weight gain.[4] It can be explained by the restraint model, which explains BED as anxiety from body weight. It leads to an ongoing cycle of restraint and binge eating. People who seek weight loss are implemented dietary restraint, which may not be able to accomplish without experiencing binge eating episodes.[12] An increase in adversity childhood events may also lead to binge-eating episodes. [5] Social factors such as idealization of thinness cause BED, indirectly caused by increasing sensitivity to the ideal of thinness or by influencing choices in the peer environment.[6] Parental education, parental pressure, and behavior change, such as excessive control over a child's diet, are also linked to the child's eating behavior. [7] BED may also be linked to other mental disorders including anxiety and depression. [11] Those phenomena can be explained by the affect regulation model and escape theory. The affect regulation model suggests that an increase in negative emotions causes overeating, then people use binge eating to relieve negative emotions and find food as

afenghstina0303@gmail.com 
comfort and distraction. Escape theory explains that people use binge eating as a strategy to distract attention rather than solve the stressors. This is thought to be an attempt to concentrate on the current stimulus, such as the experience of eating rather than current feelings. [12] To be more specific about stress, stress increases the amount of high-sugar and fat food the people eat but does not increase the amount of low-sugar and low-fat food, resulting in an indication of the effect of stress on the amount of food eaten.[16]

In a case study, the researchers found a more complex relationship between emotional states and binge eating. Some patients with binge eating may be termed as "mood intolerance" that is of adverse mood states, such as anger, depression even excitement who is intolerant of all the intense emotions. These patients often used "dysfunctional mood modulatory behavior"(self-injury, taking psychoactive substances, binge eating, vomiting, and intense exercising) to dissipating the initial emotions.[21]

There are also associations between binge eating at 14 years old and future health-related outcomes at ages 16 and 19 years. A population-based study finds that dieting was associated with the development of both binge eating and purging. Dieting, high levels of neuroticism (susceptibility to mood, depression, worry anxiety, and hypersensitivity), behavioral problems, personality profiles, and deliberate self-harm at 14 years were associated with future binge eating at 16 or 19 years. Binge eating at 14 years was associated with future purging at 16 or 19 years. [9]

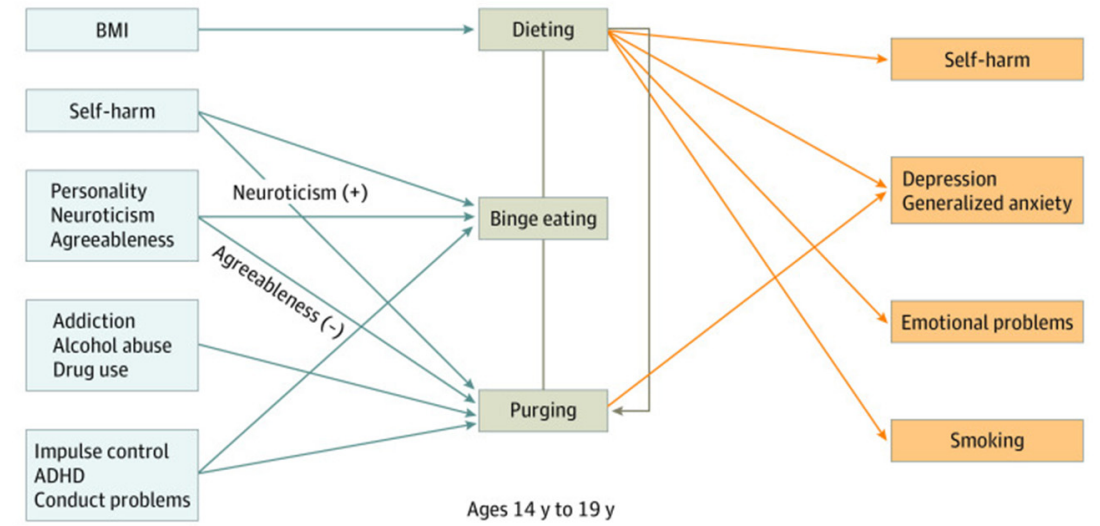

Fig 1. Time-Varying Associations and Outcomes of Disordered Eating Behaviors (DEBs) From 14 to 19 Years[9]

In China, negative emotions are still associated with binge eating. Family relationships, stress, and social standards that bring negative emotions are all factors that influence binge eating among teenagers. Chinese teenagers also lack education about healthy eating, which results in bad eating habits and leads to overeating. [18] Study shows the higher the degree of anxiety and difficulty of regulating emotions. Chinese college students tend to eat more food. [14] Girls have larger tendencies of binge eating than boys, but the severity of the behavior varies by grade. High school and college students are more likely to binge eating than middle school students due to the high pressure in upper grade.

Influenced by the aesthetics of "thin privilege", some Chinese teenagers, especially females, have taken to dieting to lose weight. However, most dieters do not lose weight, and extreme dieters suffer from eating disorders as a result. The study investigated the dieting status of college students in Yunnan province and further explored the mechanism of the participants' dieting cognition and behavior tendency on their self-evaluation and mood. The results show that more girls tend to be in dieting than boys, and perceived ineffectiveness of dieting in behavior can negatively affect emotions through self-evaluation. [17]

A study has found a significant correlation between bullying, perceived stress, and eating disorders among Chinese college students. Being bullied refers to an individual's long-term or repeated aggressive behavior toward others or groups. Being bullied would affect the individual's mental health and may lead to extreme behavior such as suicide, aggression, etc. The incidence of bullying in the study was $29.95 \%$, the stress level was medium level, and the eating disorder was slightly lower than medium level, among which $24.73 \%$ were inclined to BED. [13]

\section{Treatment}

\subsection{Psychotherapies}

Treatment for BED largely targets the behavioral, psychological, and physical outcomes related to the disorder. Cognitive behavior therapy (CBT) is one of the common and effective interventions for BED. It focuses on identifying relationships about thoughts and behaviors. The purpose of CBT is to reduce negative emotions and unhealthy behavior patterns by developing more positive responses to the factors that trigger BED. CBT can be delivered in various forms according to the level of therapist involvement, such as self-help CBT, guided selfhelp CBT, or structured self-help CBT. The patient can follow a treatment bool without the help of a facilitator in self-help CBT, which can help patients to decreased dietary restraint scores and shape concern. However, selfhelp CBT can not reduce days with binge eating and depressive symptoms. Therapist-led CBT may decrease days with binge eating, reduce depression, concern with 
weight and eating. The American Psychological Association and the National Institute for Health and Care Excellence recommend both therapist-led and self-help forms of CBT for treating the behavioral and psychological symptoms of the disorder as the first-line approach.

As, potential psychological alternatives for BED, Interpersonal psychotherapy (IPT) is based on the belief in binge eating and is used as a coping mechanism to avoid the negative effects of low interpersonal social function. IPT focus on identifying and changing the quality of patients' interpersonal functioning to help reduce their negative emotions and unhealthy behaviors. Compared CBT to IBT, they showed similar efficacy but CBT works more rapidly. IBT is still a good second approach to improve the condition of BED. However, it needs more research to matching therapies with different phenotypes of BED.[19,20]

Behavioral weight loss (BWL), combined with various behavioral strategies to promote weight loss(e.g. restricting calorie intake), is usually recommended for patients with excess weight that is common among the Binge eating population. The patients who experienced BWL have greater BMI loss than patients who experienced CBT, but BWL is not successful at achieving weight loss compared to CBT. Therefore, the intervention of BWL's effect on binge eating maybe less durable than CBT. [19,20]

Dialectical behavior therapy (DBT) focuses on increasing mindfulness and developing skills to help patients improve emotion regulation and interpersonal relationships, which may be one of the treatments for BED in elderly patients. Some research found that patients had great improvements in binge eating, body image, eating habits, and negative emotions after experiencing DBT.[20,22]

\subsection{Pharmacological treatments}

Pharmacotherapy has a significant effect on reducing the days with BED. Common forms of pharmacotherapy include antidepressants, anticonvulsants, antiobesity agents, and central nervous stimulants. Antidepressants work by selectively inhibiting the reuptake of neurotransmitters that affect mood and emotions. (e.g. dopamine and serotonin) These medicines can help patients to improve their mood.

Common antidepressants include bupropion, citalopram, and desipramine. Antiobesity agents are used to treating obesity. The common medicine includes orlistat that decreases fat absorption in the gut. Central nervous stimulants are usually used to accelerate mental and physical processes. Lisdexamfetamine, currently approved drug by the U.S. Food and Drug Administration for treating $\mathrm{BED}$, is under this class. It also has a strong effect in reducing obsessions, compulsions, and weight. Compared with placebo, lisdexamfetamine and antidepressants can result in greater abstinence from binge eating. Also, antidepressants are more effective in reducing depressive symptoms, and lisdexamfetamine showed a greater weight loss than placebo. Nevertheless, there are some potential side-effects that are common, including insomnia, sleep disturbances, headaches, gastrointestinal upset, and sympathetic nervous system arousal. The use of medicine should be monitored closely.[19,20] Besides Psychotherapies and Pharmacological treatments, several lines of evidence suggest that Virtual Reality (VR) has a potential utility in eating disorders, improving motivation for change, selfesteem, body image disturbances, and reducing binge eating behavior.

BED is a growing threat to the physical and mental health of teenagers in China. In addition to the treatment mentioned earlier, research also shows some special treatment for China. The study indicates that Sandplay therapy (a therapeutic intervention that reflects a person's inner thoughts) may be an effective treatment for females with binge eating disorder, although more data needed to be examined to validate. [25] Chinese high school students lack knowledge about eating quality and nutrition structure. The ignorance of the importance of eating habits is one of the key factors that causing bad eating behaviors. Educating the knowledge of nutrition can help teens reduce the days with BED. [24] Hatha Yoga can also reduce the overeating behavior of female college students, especially sustainable improvement of binge eating disorder.[26]

A significant number of people with BED have limited treatment options. The lack of specialized institutions and treatment resources, as well as the long-distance treatment facilities for people living in remote and rural areas, are often the reasons for insufficient care. Some researchers are trying to create Internet-based self-guidance (GSH) programs to fill this gap.[30] Researchers are also currently exploring a novel combination treatment approach adapted from physical exercise-and dietary therapy (PED-t). [31]

\section{Discussion}

People with BED underestimate the severity of their illness and therefore delay seeking professional help. In addition, fewer than twenty percent of the patients actually sought professional help and only 50 percent responded to current treatments, such as cognitive-behavioral therapy (CBT). The strategies for the diagnosis and treatment deserve to be further explored. Psychotherapy serves as a first-line intervention that decreased the frequency of binge eating episodes, even though their effectiveness in weight loss needs to be explored more. Behavioral and self-help interventions are effective in patients with low psychopathological characteristics. CBT and IPT decreased the frequency of binge eating episodes up to one year after the end of treatment. BWLT has been shown to be effective in reducing binge eating and weight loss, but it seems difficult to sustain over time.

However, there are no conclusions for follow-ups beyond one year due to few studies so the persistence of efficacy benefits beyond active treatment could not be evaluated. Pharmacotherapy plays an important role, but data are still limited due to the small sample size and short follow-up time. Medicine like SSRI and lisdexamfetamine 
result in remission of binge eating episodes, but the effect beyond the end of treatment is unknown.

BED requires longer, more structured follow-up studies to reveal adverse effects, cost-effectiveness, the effect of treatments for children and adolescents, and the effect of treatments on quality of life in the long term. BED in China also needs to have more data and studies to qualified the treatments. Few studies discuss the factors of BED and almost nothing for Pharmacological treatments. For adolescent binge eating disorders, there are few established treatments, and those that do exist have reported remission rates of between 30 and $40 \%$. It is necessary to develop and implement new treatments. Researchers can explore more Family-Based Treatment for teenagers, and more generally in terms of the least intensive treatment environment. The evidence base for the efficacy of almost all Pharmacological treatments, psychotherapies, and combination therapies consists of only small samples or single studies that are radically different in approach. Therefore, how to combine psychotherapy, behavioral therapy and medication for BED needs further studies. More efficacy comparative trials need to determine the equivalence or non-inferiority of treatments. Given the high levels of association between BED and negative emotions, future studies can determine whether certain treatments are better suited for teenagers in China. [20,27,28,29]

\section{Conclusion}

Binge eating disorder is a clinical syndrome recently listed as a separate diagnosis in the DSM-5. Individuals affected by BED show a lower quality of life and perceived health and higher psychological distress compared to non-obese individuals. Teenagers often experience stress from family, school, and society, leading to negative emotions and BED. Especially in China, more girls tend to be under pressure from beauty standards. In China, other causes like bullying often lead teenagers to BED. Treatment for BED is complex, with both clinical and psychological causes, as well as high dropout rates and uncertainty about achieving goals. Common treatments are separated by medication and psychotherapy. There will be further research into treatment options and therapies for BED.

\section{Acknowledgment}

Throughout the writing of this dissertation, I have received a great deal of support and assistance. I would first like to thank Professor Chiang Shan Li who teaches me lots of valuable information about Brain, Behavior, and Psychopathology. It leads me to start a plan to publish this paper. I would like to acknowledge my teacher assistant Yuanyuan Dong and Jiaqiong who give me invaluable experience in formulating the research questions and methodology. Their guidance helped me to choose the right direction and made my work to a higher level. In addition, I would like to thank my parents for their sympathetic ear. I can not finish this essay without their emotional support.

\section{References}

1. Berkman ND, Brownley KA, Peat CM, et al. Management and Outcomes of Binge-Eating Disorder [Internet]. Rockville (MD): Agency for Healthcare Research and Quality (US); 2015 Dec. (Comparative Effectiveness Reviews, No. 160.) Table 1, DSM-IV and DSM-5 diagnostic criteria for binge-eating disorder. Available from: https://www.ncbi.nlm.nih.gov/books/NBK338301/ta ble/introduction.t1/

2. Smink FR, van Hoeken D, Hoek HW. Epidemiology of eating disorders: incidence, prevalence and mortality rates. Curr Psychiatry Rep. 2012 Aug;14(4):406-14. doi: 10.1007/s11920-012-0282-y. PMID: 22644309; PMCID: PMC3409365.

3. Jung JY, Kim KH, Woo HY, Shin DW, Shin YC, Oh $\mathrm{KS}$, Shin EH, Lim SW. Binge eating is associated with trait anxiety in Korean adolescent girls: a cross sectional study. BMC Womens Health. 2017 Jan 21;17(1):8. doi: 10.1186/s12905-017-0364-4. PMID: 28109277; PMCID: PMC5251209.

4. Field AE, Austin SB, Taylor CB, Malspeis S, Rosner B, Rockett HR, Gillman MW, Colditz GA. Relation between dieting and weight change among preadolescents and adolescents. Pediatrics. 2003 Oct;112(4):900-6. doi: 10.1542/peds.112.4.900. PMID: 14523184.

5. McKnight Investigators. Risk factors for the onset of eating disorders in adolescent girls: results of the McKnight longitudinal risk factor study. Am J Psychiatry. 2003 Feb;160(2):248-54. doi: 10.1176/ajp.160.2.248. Erratum in: Am J Psychiatry. 2003 May;160(5):1024. PMID: 12562570.

6. Keel PK, Forney KJ. Psychosocial risk factors for eating disorders. Int J Eat Disord. 2013 Jul;46(5):4339. doi: 10.1002/eat.22094. PMID: 23658086.

7. Agras WS, Bryson S, Hammer LD, Kraemer HC. Childhood risk factors for thin body preoccupation and social pressure to be thin. J Am Acad Child Adolesc Psychiatry. 2007 Feb;46(2):171-8. doi: 10.1097/chi.0b013e31802bd997. PMID: 17242620.

8. Killen JD, Taylor CB, Hayward C, Wilson DM, Haydel KF, Hammer LD, Simmonds B, Robinson TN, Litt I, Varady A, et al. Pursuit of thinness and onset of eating disorder symptoms in a community sample of adolescent girls: a three-year prospective analysis. Int J Eat Disord. 1994 Nov;16(3):227-38. doi: 10.1002/1098-108x(199411)16:3<227::aideat2260160303>3.0.co;2-1. PMID: 7833956.

9. Robinson L, Zhang Z, Jia T, Bobou M, Roach A, Campbell I, Irish M, Quinlan EB, Tay N, Barker ED, Banaschewski T, Bokde ALW, Grigis A, Garavan H, Heinz A, Ittermann B, Martinot JL, Stringaris A, Penttilä J, van Noort B, Grimmer Y, Martinot MP, Insensee C, Becker A, Nees F, Orfanos DP, Paus T, Poustka L, Hohmann S, Fröhner JH, Smolka MN, Walter H, Whelan R, Schumann G, Schmidt U, Desrivières S; IMAGEN Consortium. Association of Genetic and Phenotypic Assessments With Onset of 
Disordered Eating Behaviors and Comorbid Mental Health Problems Among Adolescents. JAMA Netw Open. 2020 Dec 1;3(12):e2026874. doi: 10.1001/jamanetworkopen.2020.26874. PMID: 33263759; PMCID: PMC7711322.

10. McCabe MP, Ricciardelli LA, Finemore J. The role of puberty, media and popularity with peers on strategies to increase weight, decrease weight and increase muscle tone among adolescent boys and girls. J Psychosom Res. 2002 Mar;52(3):145-53. doi: 10.1016/s0022-3999(01)00272-0. PMID: 11897233.

11. McClelland J, Robinson L, Potterton R, Mountford V, Schmidt U. Symptom trajectories into eating disorders: A systematic review of longitudinal, nonclinical studies in children/adolescents. Eur Psychiatry. 2020 May 26;63(1):e60. doi: 10.1192/j.eurpsy.2020.55. PMID: 32450945; PMCID: PMC7355161.

12. McCuen-Wurst $\mathrm{C}$, Ruggieri $\mathrm{M}$, Allison KC. Disordered eating and obesity: associations between binge-eating disorder, night-eating syndrome, and weight-related comorbidities. Ann N Y Acad Sci. 2018 Jan;1411(1):96-105. doi: 10.1111/nyas.13467. Epub 2017 Oct 16. PMID: 29044551; PMCID: PMC5788730.

13. Mengyang Wang.The Influence of Bullying on College Students with Eating Disorders in China [D].Harbin Normal University,2020.

14. Shiyu Tang.The relationship between attachment, emotional regulation difficulties and food cravings among adult college students [D].Shanghai Normal Univeristy, 2020.

15. Yuanjie Wang.The influence of psychological stress on abnormal eating behavior of adolescents[D]. WUhan Sports University,2020.

16. Wei Qi.Why does stress lead to increased eating[D].East China Normal University,2019.

17. Lingyu Deng. The Relationship between Dieting Status and Emotion of College Dieters: The Mediating Role of Self-evaluation[D].Yunnan Normal University,2017.

18. Shengqiang Deng.Investigation on Dietary Behavior of Senior High School Students in Yangzhou and Changzhou City, Jiangsu Province[D].Yangzhou University,2013.

19. McCuen-Wurst C, Ruggieri M, Allison KC. Disordered eating and obesity: associations between binge-eating disorder, night-eating syndrome, and weight-related comorbidities. Ann N Y Acad Sci. 2018 Jan;1411(1):96-105. doi: 10.1111/nyas.13467. Epub 2017 Oct 16. PMID: 29044551; PMCID: PMC5788730.

20. Brownley KA, Berkman ND, Peat CM, Lohr KN, Cullen KE, Bann CM, Bulik CM. Binge-Eating Disorder in Adults: A Systematic Review and Metaanalysis. Ann Intern Med. 2016 Sep 20;165(6):40920. doi: 10.7326/M15-2455. Epub 2016 Jun 28. PMID: 27367316; PMCID: PMC5637727.
21. Cooper Z, Fairburn CG. The Evolution of "Enhanced" Cognitive Behavior Therapy for Eating Disorders: Learning From Treatment Nonresponse. Cogn Behav Pract. $2011 \quad$ Aug;18(3):394-402. doi: 10.1016/j.cbpra.2010.07.007. PMID: 23814455; PMCID: PMC3695554.

22. Chapman AL. Dialectical behavior therapy: current indications and unique elements. Psychiatry (Edgmont). 2006 Sep;3(9):62-8. PMID: 20975829; PMCID: PMC2963469.

23. de Carvalho MR, Dias TRS, Duchesne M, Nardi AE, Appolinario JC. Virtual Reality as a Promising Strategy in the Assessment and Treatment of Bulimia Nervosa and Binge Eating Disorder: A Systematic Review. Behav Sci (Basel). 2017 Jul 9;7(3):43. doi: 10.3390/bs7030043. PMID: 28698483; PMCID: PMC5618051.

24. Anxian Hou. Research on eating disorder tendency and physical condition and its relationship among junior high school students in Beijing[D].Beijing Sport University,2016.

25. Aili Wang.Study on the curative effect of sandplay therapy on female binge eating disorder and atypical binge eating disorder[D].East China Normal University,2011.

26. Hongmei Li.Effects of Hatha Yoga Intervention on Body Composition and Cardiopulmonary Fitness of Female College Students with High Risk of Eating Disorder [D].Beijing Sport University,2019.

27. Amianto F, Ottone L, Abbate Daga G, Fassino S. Binge-eating disorder diagnosis and treatment: a recap in front of DSM-5. BMC Psychiatry. 2015 Apr 3;15:70. doi: 10.1186/s12888-015-0445-6. PMID: 25885566; PMCID: PMC4397811.

28. Swedish Council on Health Technology Assessment. Treatment for Binge Eating Disorder [Internet]. Stockholm: Swedish Council on Health Technology Assessment (SBU); 2016 Apr. SBU Assessments No. 248. PMID: 28876759.

29. Palavras MA, Hay P, Filho CA, Claudino A. The Efficacy of Psychological Therapies in Reducing Weight and Binge Eating in People with Bulimia Nervosa and Binge Eating Disorder Who Are Overweight or Obese-A Critical Synthesis and MetaAnalyses. Nutrients. 2017 Mar 17;9(3):299. doi: 10.3390/nu9030299. PMID: 28304341; PMCID: PMC5372962.

30. Munsch S, Wyssen A, Vanhulst P, Lalanne D, Steinemann ST, Tuch A. Binge-eating disorder treatment goes online - feasibility, usability, and treatment outcome of an Internet-based treatment for binge-eating disorder: study protocol for a three-arm randomized controlled trial including an immediate treatment, a waitlist, and a placebo control group. Trials. 2019 Feb 13;20(1):128. doi: 10.1186/s13063019-3192-z. PMID: 30760299; PMCID: PMC6375147.

31. Mathisen TF, Rosenvinge JH, Pettersen G, Friborg O, Vrabel K, Bratland-Sanda S, Svendsen M, Stensrud T, 
Bakland M, Wynn R, Sundgot-Borgen J. The PED-t trial protocol: The effect of physical exercise -and dietary therapy compared with cognitive behavior therapy in treatment of bulimia nervosa and binge eating disorder. BMC Psychiatry. 2017 May 12;17(1):180. doi: 10.1186/s12888-017-1312-4. PMID: 28494809; PMCID: PMC5427572. 